RÉSUMÉ

\section{HIt and run}
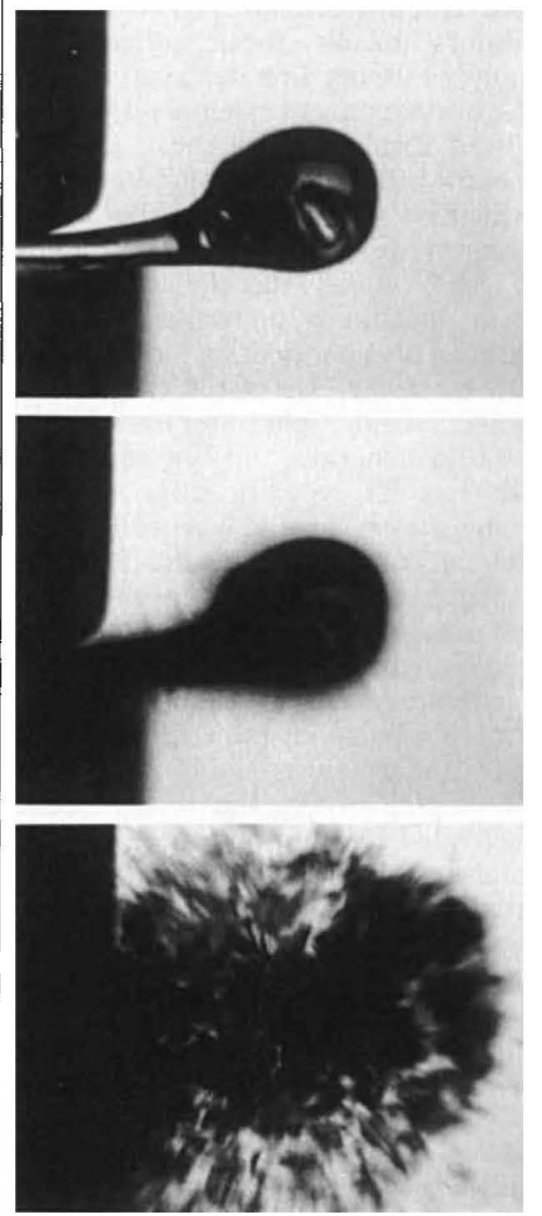

Peter Carey's novel Oscar and Lucinda includes an eerie description of the nearmagical properties of 'Prince Rupert's drops'. Named for a seventeenth-century Bavarian prince, these tadpole-shaped glass droplets can be formed by dripping molten glass into water. The rapid cooling produces a drop whose surface is in compression but whose interior is in tension (something of the same trick is used to manufacture safety glass for car windscreens). Even a heavy hammer blow to the head of such a droplet will not break it - but snip off the tail, and the entire droplet shatters in an instant, as this sequence of photographs shows; the final frame is taken less than $0.5 \mathrm{~ms}$ after breaking the tail (S. Chandrasekar and M. M. Chaudhri Phil. Mag. B70, 1195$1218 ; 1994)$. To determine how it happens, the authors detonated tiny explosive charges on the tails of droplets suspended in water, then used highspeed photography to follow developments. Shadowgraphs of the initially transparent droplets revealed the 'front' of an ominously dark region speeding from tail to head at up to $1.9 \mathrm{~km}$ $\mathrm{s}^{-1}$. They conclude from this and electron micrographs of the remains that rapidly bifurcating cracks propagate through the highly stressed interior until the entire droplet is fragmented, whereupon the minute shards fly apart at up to $25 \mathrm{~m} \mathrm{~s}^{-1}$. \section{Modulation by instruction}

\author{
Michael Posner
}

FOR 100 years it has been assumed that attention - that is, voluntary concentration on an actual or expected stimulus somehow improves processing of the selected neural message. Some theories have held that this modulation occurs only late in stimulus processing, in areas of the brain most likely to generate responses; others, that modulation takes place early on, at the sensory-input stage ${ }^{1}$.

On page 249 of this issue ${ }^{2}$, in a paper describing positron emission tomography (PET) studies of blood flow in the brain, Drevets et al. show clear local changes in brain activity within the sensory areas that process the attended signals. This finding supports other PET studies that have shown that blood flow is modulated within extrastriate visual areas during attention $^{3,4}$. Although blood flow is an indirect measure, there is strong evidence that changes in blood flow do indeed reflect local neuronal activity ${ }^{5}$. So these PET studies provide us with clear indications that instructions to expect a target can produce voluntary, central modulation of activity within sensory areas.

The next question to ask is how attention modulates sensory input. For example, does it act to amplify the selected message, to suppress competing messages or through some combination of the two? Within the experimental conditions of their study, Drevets et al. provide an unusually clear answer. They subtract measurements in a resting state, in which subjects do not prepare for a stimulus, from those made under conditions in which subjects prepare for a somatosensory stimulus that might or might not be painful. In fact, subjects do not receive any stimuli during the experimental scan, but merely prepare for one. Blood flow shows a clear decrease when expecting a stimulus. This decrease is in non-attended somatosensory areas surrounding, but not including, the area at which the stimulus was expected. As the authors indicate, this result clearly supports the importance of suppression of potentially competing neuronal activity in improving processing of the attended signal.

Under somewhat different conditions, however, it is easy to find evidence that blood flow increases during attention to sensory events. Indeed both of the PET studies cited above $e^{3,4}$ found increasing blood flow in sensory areas when subjects actually processed attended target stimuli (for example by making a response to them). In addition, last month Heinze $e t$ $a l{ }^{6}$ showed clear increases in blood flow in the visual object perception pathway when subjects attended to a visual target; this increased blood flow was also re- flected in increased electrical activity, recorded from scalp electrodes, apparently related to activity in areas close to those showing increased blood flow. So readers of these and other papers in the field must pay careful attention to the exact experimental task and methods used in order to work out the details of how attention actually influences sensory processing.

Two difficulties in interpreting PET studies must be taken into consideration. First, changes in blood flow reflect both excitatory and inhibitory synaptic activity $^{7}$; so it remains to be discovered exactly how suppression in blood flow in areas surrounding the focus of attention influences the activity of cells coding input to that area. Second, the PET results represent an average of 40 seconds in which subjects either await a signal or are passive. To determine the time over which attention has an influence, it is possible to compare electrical recordings when subjects attend to actual targets with those obtained without attention. These eventrelated potentials show that attention produces both increases and decreases in electrical activity within a few hundred milliseconds ${ }^{8}$ of each other. For example, recordings from scalp electrodes have shown that early parts of the event-related potential that occur after about 100 milliseconds are suppressed at unattended locations, whereas later components are increased at attended locations ${ }^{8}$. Cellular studies suggest that initial increases in activity at the attended location are suppressed within 100 milliseconds ${ }^{9}$. From these findings, it seems that attending to targets, at least within the visual system, involves a complex of changes that occur rather rapidly. The average of 40 seconds may only provide a crude net effect of what is actually taking place.

A central feature of Drevets and colleagues' study is that no stimuli actually occurred during the scan. Instead, subjects were merely asked to attend in case they occurred. These are the conditions of high vigilance to external events that have been studied extensively for auditory and visual signals ${ }^{10}$. During a warning interval there is often widespread suppression of both physical and mental activity ${ }^{1}$. For example, during preparation for a stimulus, heart rate slows and bodily and mental activity that might compete with a target is suppressed. Such suppression has usually been interpreted as reducing competition, so that a potential target will have maximal influence ${ }^{1}$. However, there is no evidence in these studies that the suppression is so exquisitely linked to the sensory areas surrounding the attended location as re- 